\title{
Natural Teeth-A Novel Biomaterial for Bone Regenration
}

\author{
Navneet Kaur* \\ Department of Periodontics, Faculty of dentistry, Baba Farid University of Health sciences, India
}

*Corresponding author: Navneet Kaur, Department of Periodontics, Faculty of dentistry, National Dental College \& Hospital Derabassi Punjab, India.
Received Date: March 01, 2021

Published Date: March 15, 2021

\begin{abstract}
Nowadays, various bone graft materials are developed due to advanced bio graft material development technologies; as a result, dento alveolar surgery can handle difficult osseous defects with various methods in the placement of implant, periodontal surgery, and maxillofacial surgery. The auto-tooth bone graft material is a system that treats patients by manufacturing bone graft material from their own extracted teeth. Auto-tooth bone graft material is useful as it supports excellent bone regeneration capacity and minimizes the possibility of foreign body reaction, genetic diseases and eliminating the risk of an immune reaction that may lead to the rejection. The emergence of autogenous tooth bone graft based on the principles of Endogenous Regenerative Technology (ERT) promotes not only the natural healing process but also reconstruction of lost tissues by body's inherent capabilities. It is therefore, difficult to obtain bone graft material with the advantages of autogenous bone but not its disadvantages. Thus, the search for newer, near ideal biomaterials and procedures as well as recent application of tooth as a bone graft material may continue to be a challenge in the field of regenerative periodontics.
\end{abstract}

Keywords: Biomaterial; Osteoconduction; Osteoinduction; Bone graft; Bone morphogenetic protein

\section{Introduction}

Bone graft and Bone substitutes are developed to reconstruct the bony defects which is rich in growth factors and BMPs. Autologous bone graft has osteogenic, osteoconductive and osteoinductive properties and therefore considered as a gold standard in bone reaeration. Researchers and Clinicians choose autogenous tooth bone graft which allows both formation of new bone and is steadily observed to be replaced by bone without compromising on the bone regeneration capacity. There is a suggestion that teeth can also be used as a bone graft material which may act as a novel biomaterial for all the bony defects. Dentin and bone are similar mineralized tissues in terms of chemical composition such as hydroxyapatite. Autogenous tooth bone graft material (AutoBT) was first developed in 2008 and has been used mainly for guided bone regeneration to supplement dental implants [1]. It is a bone graft material that is obtained using extracted teeth. The amount of bone graft obtained depends on the condition of discarded teeth and its histological outcomes are similar to autogenous bone grafts [2]. The objective is to assess the utility of autologous dentin for

1. Its efficiency as a graft material in implantology.

2. Preservation and augmentation of alveolar ridge. 3. An alternate to other synthetic bone graft material.

\section{Natural teeth as bone substitute}

Dentin has been used as a bone substitute and considered as an ideal bioactive material for hard tissue regeneration. Dentin and alveolar bone are made up of $65 \%$ inorganic and $35 \%$ organic components. Because of the following features it may be considered as natural biomaterial and potentially useful in clinics [3].

1. Rich source of stem cells, matrix, trace metal ions, and growth factors 
2. Also contains variety of proteins such as osteopontin, bone sialoprotein, dentin sialoprotein and osteocalcin.

3. Dentin contains some growth factors such as TGF- $\beta$, BMP IGF-II.

\section{Historical View}

Hegedeus [4] was the first person to report the use of Autogenous bone grafts in the reconstruction of periodontal defect. He reported success in 6 cases by transplanting Autogenous bone from the tibia to the jaws to treat "advanced pyorrhea"[4]. Ike and Urist suggested recycled root dentin prepared from extracted teeth for use as a carrier of Recombinant human BMP-2 (rh-BMP2) because it induces new bone formation in the periodontium.

Kim is the pioneer for doing research on natural tooth and used as a bone graft material. Kim et al., in 1993, conducted basic studies such as component analysis, research through EM, and production of autogenous bone graft material after incinerating human teeth at a high temp. of $135^{\circ} \mathrm{C}$ and then pulverizing to a particle size of $0.149 \mathrm{~mm}[5,6]$. In 2008, development of an autogenous tooth bone graft material (AutoBT; Korea Tooth Bank Co., Seoul, Korea) from extracted teeth prepared as powder and grafted it to the donor patient himself [3]. Mineral components of autogenous tooth bone graft materials consist of 4 stages of calcium phosphate i.e., HA, TCP, OCP and ACP. Under SEM examination, HA crystalline structures and collagen fibers around the dentinal tubules were detected.

Tooth Banking services such as Bioeden, Dentcell, Mothercell, Future Health bio bank etc. and in Korea, the Korea Tooth Bank performed bio-recycling of 38,000 patient own teeth from January 2009 until October 2012.

\section{Osteoinductive and Osteoconductive Property}

Various authors did number of researches on the property of osteoconductive and Osteoinductive of Autogenous bone graft material. Studies showed that autogenous DDM possessed osteoconductive and osteoinductive properties similar to the one present in bone. According to biochemical and histomorphic analysis of bone and cartilage induced by human DDM and BMP-2 researchers concluded that human DDM of vital teeth and BMP-2 enhances and accelerate bone formation in DDM carrier system.

Nampo et al used extracted teeth as graft material for alveolar repair and concluded that dentin has high affinity and marked osteoconductive effect on jaw bones [7]. Bessho, et al. [8] successfully isolated BMP from demineralized bone and dentin of extracted teeth from rabbits. Purified BMP is homogenous and induced bone formation in 3 weeks when implanted in the muscle pouches in wistar rats [8].

\section{Preparation of Autogenous Bone Graft Material}

The extracted teeth are cleaned. Amalgam, composites, and endodontic filling material are removed from the teeth with a diamond bur, under water cooling, and with a turbine. The tooth fragments are then thoroughly dried and placed in the chamber. The chamber is tightly closed. The "grind" setting is adjusted to 3 seconds and "sort" to 20 seconds before the grinding process begins. The unit sorts the granules into two filtration chambers. Dentin grinder grinds the tooth material with a particle size in between 300-1200microns. The particle size less than 300 microns is considered as an efficient size for bone grafting. The ground and filtered material is immersed in a cleanser solution for 10 minutes. The cleanser, which s bactericidal, consists of sodium hydroxide with $20 \%$ ethanol. The resulting particulate is carefully dried, before being neutralized in a buffering solution for $3 \mathrm{~min}$. and then again dried with sterile swabs. A third optional stage, involving the use of EDTA solution for 1 minute, can be added between cleansing and neutralizing if so desired. After the tooth has been ground and processed, the resulting "sticky bone" can be used as graft in the alveolus.

\section{Forms of autogenous bone graft}

1. Block type: This type graft material demonstrates Osteoinduction capacity through blood wettability and also has osteoconduction capacity though space maintenance capability along with creeping substitution.

2. Powder type: it is supplied based on various size of particles, porosity, blood wettability, osteoconduction, Osteoinduction and creeping substitution.

\section{Bone graft chips.}

All these forms can be used for preservation of extraction socket, restoration of perforated sinus membrane, augmentation procedures and also in periodontal defects. Powder type (75\%) is most commonly used form than block type. Combination of powder and block type is only $9 \%$ and used in clinical applications [9].

\section{Advantages}

1. Promote healing of socket/wound.

2. Quick Bone growth within 6-8 weeks.

3. Graft can be ready within 15 mins.

4. No risk of immune reaction.

5. An ideal scaffold for stem cells and bone growth factor

6. Safe and effective bone graft material.

\section{Limitations}

1. Sacrifice the healthy tooth to obtain a graft.

2. Chances of graft resorption.

3. Limited availability of autogenous bone graft material. 


\section{Clinical Applications [10]}

1. Tooth socket preservation: Auto tooth bone graft material may be placed after 12 weeks in the extraction socket to secure enough edentulous bone to reserve alveolar bone and place implant if necessary, since tooth extraction may be performed due to periodontal defect, injury, root fracture, or root rest.

2. Distoangular impaction of third molar.

3. Autogenous bone graft in Implant surgery: Auto tooth bone graft material may be placed with an implant in case of osseous defect larger than $2 \mathrm{~mm}$ vertically or horizontally around the placed implant. If the amount of graft material is insufficient and for additional volume it can be mixed with other bone graft material.

4. Guided Bone regeneration.

5. Mandibular ridge augmentation: If the vertical and horizontal diameters of alveolar bone is shorter than $3 \mathrm{~mm}$, block type auto tooth graft material can be placed to increase bone mass.

6. Grafting of maxillary sinus (Sinus lifting): used for patient whose edentulous bone is less than $10 \mathrm{~mm}$ height from the sinus floor via lateral or crestal approach. Only maxillary bone graft may be performed, and implant is placed after bone grafting in cases securing the initial stabilization of the implant is difficult due to the height of the edentulous bone.

7. Graft in tumour resection

8. Cyst enucleation

Kim, et al. $[10,11]$ have con ducted large number of studies and was successful in finding out that ADDM can be used for alveolar ridge augmentation, tooth socket preservation and sinus lifts $[10,11]$. Lee, et al. [12] concluded after comparing the efficiency of autogenous DDM and other bone graft materials used in sinus bone graft surgeries; after 4 months there was favourable with faster rate and superior quality of bone formation [12]. Jiang et al in 2003 have showed that autogenous DDM may prosper in the future endodontic world as an apexification material and as a permanent root canal filling material as well [13].

\section{Conclusion}

A revolutionary bone graft material gaining popularity in the regeneration field of dentistry. Although the selection of graft materials should be dictated by the extent of ridge defects and the surgical procedure, tooth derived bone graft may be considered as a valuable alternative given its autogenous origin and positive clinical and histological results when teeth extraction is indispensable.

\section{Acknowledgement}

None

\section{Conflict of Interest}

No Conflict of Interest.

\section{References}

1. Kim YK, Lee JH, Um IW, Cho WJ (2016) Guided Bone Regeneration Using Demineralized Dentin Matrix: Long-Term Follow-Up. J Oral Maxillofac Surg 74: 515. e1-e9.

2. Jeong KI, Kim SG, Kim YK, Oh JS, Jeong MA, et al. (2011) Clinical study of graft materials using autogenous teeth in maxillary sinus augmentation. Implant Dent 20: 471-475.

3. Kim YK, Kim SG, Byeon JH (2010) Development of a novel bone grafting material using autogenous teeth. Oral Surg Oral Med Oral Pathol Oral Radiol Endo 109: 496-503.

4. Hegedus Z (1923) The rebuilding of the alveolar process by bone transplantation. Dent Cosmos 65: 736-742.

5. Kim YK, Yeo HH, Ryu CH, Lee HB, Byun UR, et al. (1993) An experimental study on the tissue reaction of tooth ash implanted in mandible body of the mature dog. J Korean Assoc Maxillofac Plast Reconstr Surg 15: 129-136.

6. Kim YK, Yeo HH, Yang IS, Seo JH, Cho JO (1994) Implantation of tooth ash combined with plaster of Paris: experimental study. J Korean Assoc Maxillofac Plast Reconstr Surg 16: 122-129.

7. Nampo T, Watahiki J, Enomoto A (2010) A new method for alveolar bone repair using extracted teeth for the graft material. J Periodontol 81: $1264-1272$

8. Bessho K, Tagawa T, Murata M (1990) Purification of rabbit bone morphogenetic protein derived from bone, dentin, and wound tissue after tooth extraction. J Oral Maxillofac Surg 48: 162-169.

9. Sung Min Park (2012) Clinical application of auto-tooth bone graft material. J Korean Assoc Oral Maxillofac Surg 38: 2-8.

10. Kim YK (2010) Clinical application and classification of bone graft material according to component. J Korean Dent Assoc 48: 263-274.

11. Kim YK, Kim SG, Yun PY (2014) Autogenous teeth used for bone grafting: a comparison to traditional grafting materials. Oral Surg Oral Med Oral Pathol Oral Radiol 117: e39-45.

12. Lee (2011) Histomorphometric study of sinus bone graft using various graft material. J Dent Rehabil Appl Sci 27: 141-147.

13. Jeong HR, Hwang JH, Lee JK (2011) Effectiveness of autogenous tooth bone used as a graft material for regeneration of bone in miniature pig. J Korean Assoc Oral Maxillofac Surg 37: 375-379. 